\title{
ASSOCIAÇÃO DA ATIVIDADE FÍSICA DE LAZER E DO DESLOCAMENTO OCUPACIONAL COM A CAMINHADA E O CICLISMO: UM ESTUDO TRANSVERSAL COM BRASILEIROS ADULTOS
}

\section{ASSOCIATION OF THE PHYSICAL ACTIVITY FOR LEISURE AND OCCUPATIONAL DIS- PLACEMENT WITH THE WALKING AND THE CYCLING: A CROSS-SECTIONAL STUDY WITH BRAZILIAN ADULTS}

\author{
Dartel Ferrari Lima ${ }^{a^{*}}$ \\ Orcid: http://orcid.org/0000-0002-3633-9458 \\ Lohran Anguera Lima ${ }^{b^{* *}}$ \\ Orcid: http://orcid.org/0000-0002-8303-5588 \\ Oldemar Mazzardo Júnior ${ }^{{ }^{\star}}$ \\ Orcid: http://orcid.org/0000-0001-5325-9815 \\ Adelar Aparecido Sampaio ${ }^{\mathrm{d}^{*}}$ \\ Orcid: http://orcid.org/0000-0003-4386-1364
}

Michael Pereira da Silva ${ }^{\mathrm{e}^{*}}$

Orcid: http://orcid.org/0000-0002-7021-9847

\author{
Arestides Pereira da Silva Júnior ${ }^{*}$ \\ Orcid: http://orcid.org/0000-0003-0647-1930
}

Veronica Gabriela Silva Piovani ${ }^{{ }^{*}}$

Orcid: http://orcid.org/0000-0002-4451-8229

Maria das Graças Anguera ${ }^{\mathrm{h}^{\star}}$

Orcid: http://orcid.org/0000-0002-0494-7467

dartelferrari07@gmail.com ${ }^{\mathrm{a}}$, lohranangueralima@gmail.com ${ }^{\mathrm{b}}$,prof.mazzardo@gmail.comc, adelarsampaio@hotmail.com ${ }^{\mathrm{d}}$,prof.mpsilva@outlook. com ${ }^{\mathrm{e}}$, arestidesjunior2000@yahoo.com.br ${ }^{\mathrm{f}}$, veropiovani@hotmail.comg, graca.anguera@bol.com.br ${ }^{\mathrm{h}}$

Universidade Estadual do Oeste do Paraná*, Santa Casa de Misericórdia de São Paulo. Departamento de Ortopedia e Traumatologia**

Data de Submissão :24/02/2019

Data de Aceite: 14/11/2019

\section{RESUMO}

Introdução: a caminhada e o ciclismo são os tipos principais de atividade física recreativa e de deslocamento utilizados por adultos brasileiros. Objetivo: associar, a partir de informações sociodemográficas e pessoais, a prática da atividade física recreativa e do deslocamento ativo de brasileiros com a caminhada e o ciclismo. Materiais e Métodos: estudo descritivo com amostra de adultos entrevistados pela Vigilância de Fatores de Risco e Proteção para Doenças. Crônicas por Inquérito Telefônico - Vigitel (2016). Resultados: 13.869 adultos (28,1\% de homens e 24,8\% de mulheres) relataram caminhar ou pedalar recreativamente; 9.607 adultos (20,1\% de homens e $16,8 \%$ de mulheres) e 4.487 adultos (7,3\% de homens e 9,2\% de mulheres) relataram deslocar-se a pé ou de bicicleta para o trabalho ou para a escola, respectivamente. Caminhar e pedalar se associou positivamente com maior força aos homens nos domínios recreativos e no deslocamento para o trabalho, enquanto o deslocamento para a escola se associou mais fortemente às mulheres, principalmente às residentes nas regiões Norte e Nordeste. No conjunto da amostra, houve maior associação para os participantes com idade de 35 a 54 anos, com maior escolaridade ( $\geq 12$ anos) e sem sobrepeso. A ocorrência de morbidades crônicas se associou com as atividades recreativas (Razão Chance: 1,43; IC95\%: 1,25-1,65). Conclusões: a caminhada e o ciclismo são as principais atividades recreativas para $27 \%$ da população brasileira e estão presentes no cotidiano de $8,0 \%$ da população que se desloca ativamente para o trabalho e/ou escola.

Palavras-chave: Recreação; Exercício; Estilo de vida sedentário; Promoção de saúde; Doença Crônica; Monitoramento.

\section{ABSTRACT}

Introduction: walking and cycling are the main types of physical activity as recreation and displacement performed by Brazilian adults. Objective: to associate the practice of the recreational physical activity and to the active displacement with the walking and the cycling. Materials and Methods: a descriptive study with a sample of adults interviewed by the Risk Factors and Protection for Chronic Diseases Surveillance by Telephone Survey - Vigitel (2016). Results: 12,333 adults (25.4\% men and $21.8 \%$ women) reported walking or cycling recreationally, 9,607 adults (20.1\% men and $16.8 \%$ women) and 4,487 adults (7.3\% men and 9.2\% women) respectively went on foot or by bicycle to work and to school; walking and cycling were positively associated with men in the recreational areas and on the displacement to work, while the displacement to school was stronger associated with women, especially those living in the North and Northeast. 
In the whole sample, there was a greater association for participants aged 35 to 54 years, with higher schooling $(\geq$ 12 years) and without overweight. The occurrence of chronic morbidities was only associated with recreational activities (odds ratio: 1.43; 95\%: CI 1.25-1.65). Conclusion: walking and cycling are the main recreational activities for $27 \%$ of the Brazilian population and are present in the daily life of $8.0 \%$ who actively move to work and / or to school.

Keywords: Recreation; Exercise; Sedentary Lifestyle; Health Promotion; Chronic Disease; Monitoring.

\section{Introdução}

Conhecer a participação de pessoas em atividade física (AF), especialmente no contexto da prevenção de doenças, é essencial para compreender as relações da inatividade física com o surgimento e/ou agravamento de patologias relacionadas à hipocinesia ${ }^{1}$. Também, como consequência, aspectos relacionados à prática de AF podem fornecer informações para melhor avaliar o efeito das intervenções destinadas à saúde pela promoção da AF.

Algumas mudanças das relações sociais e do próprio senso de organização social, como o processo de urbanização das grandes cidades, estão em contínuo fluxo, influenciando as dimensões do tempo e do espaço, dando um caráter rotineiro ao cotidiano das pessoas. ${ }^{2}$ Por exemplo, o desenvolvimento e a oferta de sistemas de transporte coletivo, ao mesmo tempo que facilita o deslocamento das massas urbanas, contribui para a redução do deslocamento ativo a pé ou de bicicleta.

Embora estratégias estejam em andamento para enfrentar a inatividade física, os seus efeitos permanecem ainda limitados e os resultados inacabados ${ }^{3}$, principalmente porque a relação entre a AF e a saúde está longe de ser simples ${ }^{4 ; 5}$. A compreensão da relação da dose e do efeito obtido nos diferentes domínios da AF necessita de maior entendimento, principalmente aqueles relacionados aos domínios ocupacional e do lar.

Parece pacificado, no entendimento atual, que a AF tem um limite de benefício para os vários eventos de saúde. Esses efeitos são a favor de um limiar mínimo, ou seja, o efeito é determinado conforme o volume semanal e pode ser alcançado por diferentes modos (lazer, ocupacional, deslocamento e no lar $)^{4}$. Nessa perspectiva, a Organização Mundial da Saúde (OMS) recomenda aos adultos, para obterem efeitos substanciais de saúde, o alcance mínimo de 150 minutos por semana de AF de intensidade moderada ou 75 minutos por semana de AF de intensidade vigorosa ou o equivalente de ambas as duas possibilidades ${ }^{6}$.

$\mathrm{O}$ ato de caminhar e de pedalar representa o tipo de AF de lazer e de deslocamento ativo mais utilizado pela população brasileira. A caminhada é um esforço de baixo risco cardiovascular e ortopédico, além de mecanicamente simples de ser executada por ser um movimento natural do ser humano, oferecendo boa tolerância com muito pouca necessidade de recursos técnicos e de equipamentos. No ano de 2018, a caminhada foi o principal tipo de AF recreativa para 50\% da população que reside nas capitais dos estados brasileiros e no Distrito Federal, enquanto o ciclismo alcançou, aproximadamente, $5 \%$ da população ${ }^{7}$.

A quantificação da AF praticada em seus diferentes domínios e as relações que os determinantes sociais, demográficos e individuais estabelecem com esta quantificação, possibilitam orientar as intervenções destinadas à promoção da saúde mediada pelo incentivo da participação em AF. Desse modo, este estudo, a partir de uma amostra de adultos brasileiros residentes nas capitais brasileiras e no Distrito Federal, analisou, transversalmente, a associação da prática de AF recreativa e de deslocamento ao trabalho e/ou escola com a caminhada e o ciclismo.

\section{Métodos}

Foram examinados dados transversais do sistema de Vigilância de Fatores de Risco e Proteção para Doenças Crônicas não Transmissíveis por entrevista telefônica (Vigitel) no Brasil para o ano de 2016, cuja metodologia própria pode ser acessada em publicações prévias ${ }^{7}$. Desde 2006, as 
entrevistas do sistema ocorrem anualmente e são realizadas por meio de entrevista por telefone fixo com cerca de 54.000 adultos (com idade igual ou maior do que 18 anos), com pelo menos 2.000 entrevistas em cada uma das 26 capitais brasileiras e Distrito Federal. Pesos pós-estratificação são empregados como tentativa de representar as populações com e sem telefone fixo. Em 2016, foram completadas 53.210 entrevistas.

A aprovação ética do estudo do Vigitel foi obtida pelo Comitê de Ética em Pesquisa em Seres Humanos do Ministério da Saúde do Brasil. O consentimento livre e esclarecido foi obtido oralmente no momento do contato telefônico com os entrevistados, conforme as normas éticas de investigação envolvendo seres humanos. Este estudo está dispensado de análise ética conforme preconiza a Resolução 510/2016 - CNS, por tratar de dados de acesso público, nos termos da Lei n.12.527, de 18 de novembro de $2011^{7}$.

\section{DELINEAMENTO EXPERIMENTAL}

Os participantes foram inqueridos sobre $\mathrm{o}$ principal tipo de exercício ou esporte praticado no tempo livre nos últimos três meses (apenas uma resposta foi aceita), diante uma lista com 16 opções. A caminhada (excluída aquela realizada para o deslocamento ativo para o trabalho e/ ou escola) encontrava-se na posição " 1 " e o ciclismo na posição "11". A frequência semanal e a duração diária da AF foram determinadas, respectivamente, pelas questões:

"Quantos dias por semana o(a) sr.(a) costuma praticar exercício físico ou esporte?"

"No dia em que o(a) sr.(a) pratica exercício ou esporte, quanto tempo dura esta atividade?".

Atividade com duração inferior a 10 minutos não foi considerada para efeito de cálculo.

O transporte ativo para o trabalho e para a escola foi determinado pelas respostas das questões:

"Para ir ou voltar ao seu trabalho, faz algum trajeto a pé ou de bicicleta?"

"Quanto tempo o(a) sr.(a) gasta para ir e voltar neste trajeto (a pé ou de bicicleta)?"

"Para ir ou voltar a este curso ou escola, faz algum trajeto a pé ou de bicicleta?"
"Quanto tempo o(a) sr.(a) gasta para ir e voltar neste trajeto (a pé ou de bicicleta)?”.

Foi considerado com excesso de peso o indivíduo com Índice de Massa Corporal (IMC) $\geq 25 \mathrm{~kg} / \mathrm{m} 2^{8}$, calculado a partir do peso em quilos dividido pelo quadrado da altura em metros. Foi considerado obeso o indivíduo com IMC $\geq 30$ $\mathrm{kg} / \mathrm{m}^{8}$, calculado a partir do peso (em quilos) dividido pelo quadrado da altura (em metros). Os dados antropométricos foram fornecidos pelas questões:

"O(a) sr.(a) sabe seu peso (mesmo que seja valor aproximado)?"

"O(a) sr.(a) sabe sua altura?".

As covariáveis individuais e sociodemográficas eleitas para este estudo foram: sexo (masculino e feminino); idade (agrupada em: 18-34 anos, 35-54 anos, 55-64 anos e $\geq 65$ anos de idade); escolaridade (agrupada em: 0-8 anos, 9- 11 anos e $\geq 12$ anos); estado civil (agrupado em: conjunto (união estável e casado) e avulso (solteiro, viúvo, divorciado e separado)]; morbidades referidas (diabetes, hipertensão arterial sistêmica e dislipidemia); percepção da saúde (ruim e boa) e distribuição regional dos participantes (Norte, Nordeste, Centro Oeste, Sudeste e Sul).

\section{ANÁLISE DOS DADOS}

A análise descritiva dos dados utilizou os fatores de ponderação para atribuir peso pósestratificação distintos a cada indivíduo e foi descrita utilizando tabelas com frequências relativas. O chamado método "rake" foi utilizado para estimar a população total de cada capital, com base em dados censitários do ano correspondente. Para as atividades de caminhada e de ciclismo, nos diferentes domínios e subdomínios, foram utilizadas análises com variáveis categóricas subdivididas em duas classes (ativo e inativo). $\mathrm{O}$ ativo alcançou ou ultrapassou o volume semanal de 150 minutos de $\mathrm{AF}$. $\mathrm{O}$ inativo não alcançou o volume semanal de 150 minutos de AF. O tempo gasto para caminhar e pedalar foi somado em direção à meta de 150 minutos. As variáveis investigadas foram descritas com frequências relativas. Foram realizadas regressões logísticas binárias para obtenção de Razões de Chances (RC - probabilidade de que o evento ocorra dividida 
pela probabilidade de que o evento não ocorra) e Intervalos de Confiança de 95\% (IC95\%) dos fatores associados à caminhada e ao ciclismo para os ativos nos domínios do lazer, no deslocamento para o trabalho e para a escola. Pesos amostrais e controle dos erros robustos por clusters (26 capitais mais o Distrito Federal) foram utilizados visando ajustar as estimativas de associação. $\mathrm{O}$ valor de $\mathrm{p}<0,05$ foi adotado e todas as análises foram realizadas com o software Stata $\mathrm{MP}^{\circ}$ versão 14.1 .

\section{Resultados}

Para o inquérito do Vigitel referente ao ano de 2016, inicialmente foram sorteadas 127.200 linhas telefônicas. Com base em critérios préestabelecidos, foram computadas 77.671 linhas elegíveis, das quais 53.210 responderam à entrevista; 20.258 homens e 32.952 mulheres concederam entrevistas completas. A taxa de sucesso para o inquérito foi de $68,5 \%$, oscilando entre $63,0 \%$ e $72,0 \%$, e a taxa de recusa foi de $3,8 \%$ das linhas elegíveis, variando de $1,4 \%$ a $8,4 \%$. A média da idade dos entrevistados foi de 50,3 (desvio-padrão: 18,0 anos). Para o conjunto da amostra, 13.869 adultos $(28,1 \%$ de homens e $24,8 \%$ de mulheres) declararam caminhar ou pedalar no tempo livre; 9.607 adultos $(20,1 \%$ de homens e $16,8 \%$ de mulheres) declararam se deslocar a pé ou de bicicleta para o trabalho; e 4.487 adultos (7,3\% de homens e $9,2 \%$ de mulheres) declararam se deslocar a pé ou de bicicleta para a escola.

Caminhar ou pedalar como recreação foi mais frequente em adultos de 35 a 54 anos de idade $(8,3 \%)$ e houve redução da frequência com o aumento da idade. A utilização da marcha ou da bicicleta no transporte ativo para o trabalho foi de 7,9\% na faixa de 35-54 anos de idade, com frequência cinco vezes maior àqueles com 65 anos ou mais de idade (1,6\%). A marcha e o ciclismo utilizados para o deslocamento ativo com finalidade de estudo alcançaram 3,8\% dos adultos de 18 a 34 anos de idade. Também, nos dois subdomínios do deslocamento ativo (trabalho e escola) houve decréscimo de participantes com o aumento da idade.

As duas modalidades de AF analisadas foram mais prevalentes em adultos do sexo masculino, exceto para o deslocamento à escola. No conjunto da amostra, o maior tempo de escolaridade comportou o maior percentual de marchadores e de ciclistas. $\mathrm{O}$ volume semanal de AF proporcionado pela caminhada e pelo ciclismo fez com que 37,0\% dos entrevistados ativos alcançassem a meta mínima de AF recomendada pela OMS.

A taxa de obesos ou com sobrepeso foi maior nos adultos inativos quando comparados aos seus pares ativos. Os inativos também apresentaram maior taxa de morbidades crônicas não transmissíveis e menor otimismo para avaliar o próprio estado de saúde. A região Sudeste, a mais populosa do país, apresentou a maior prevalência de deslocamento ativo para o trabalho e/ou estudo e a menor prevalência de AF recreativa (Tabela 1).

Tabela 1. Percentual ${ }^{*}$ de adultos ativos praticantes de caminhada e de ciclismo recreativo no deslocamento para o trabalho e para a escola, escore total e por sexo, segundo variáveis sociodemográficas e pessoais. Vigilância de Fatores de Risco e Proteção para Doenças Crônicas por Inquérito Telefônico (Vigitel), 2016.

\begin{tabular}{|c|c|c|c|c|c|c|c|c|c|}
\hline \multirow[b]{2}{*}{ Variáveis } & \multicolumn{3}{|c|}{ AF Lazer } & \multicolumn{3}{|c|}{ AF Trabalho } & \multicolumn{3}{|c|}{ AF Escola } \\
\hline & $\begin{array}{c}\text { Total } \\
\%\end{array}$ & $\begin{array}{c}\text { Homens } \\
\%\end{array}$ & $\begin{array}{c}\text { Mulheres } \\
\%\end{array}$ & $\begin{array}{c}\text { Total } \\
\%\end{array}$ & $\begin{array}{c}\text { Homens } \\
\%\end{array}$ & $\begin{array}{c}\text { Mulheres } \\
\%\end{array}$ & $\begin{array}{c}\text { Total } \\
\%\end{array}$ & $\begin{array}{c}\text { Homens } \\
\%\end{array}$ & $\begin{array}{c}\text { Mulheres } \\
\%\end{array}$ \\
\hline \multicolumn{10}{|c|}{ Idade (anos) } \\
\hline $18-34$ & 3,3 & 3,3 & 3,3 & 5,2 & 6,1 & 4,6 & 3,8 & 4,2 & 3,6 \\
\hline $35-54$ & 8,3 & 8,8 & 8,0 & 7,9 & 8,1 & 7,7 & 2,8 & 2,1 & 3,3 \\
\hline$\geq 65$ & 6,7 & 7,8 & 6,0 & 1,6 & 2,3 & 1,3 & 0,8 & 0,5 & 1,0 \\
\hline
\end{tabular}




\begin{tabular}{|c|c|c|c|c|c|c|c|c|c|}
\hline \multirow[b]{2}{*}{ Variáveis } & \multicolumn{3}{|c|}{ AF Lazer } & \multicolumn{3}{|c|}{ AF Trabalho } & \multicolumn{3}{|c|}{ AF Escola } \\
\hline & $\begin{array}{c}\text { Total } \\
\%\end{array}$ & $\begin{array}{c}\text { Homens } \\
\%\end{array}$ & $\begin{array}{c}\text { Mulheres } \\
\%\end{array}$ & $\begin{array}{c}\text { Total } \\
\%\end{array}$ & $\begin{array}{c}\text { Homens } \\
\%\end{array}$ & $\begin{array}{c}\text { Mulheres } \\
\%\end{array}$ & $\begin{array}{c}\text { Total } \\
\%\end{array}$ & $\begin{array}{c}\text { Homens } \\
\%\end{array}$ & $\begin{array}{c}\text { Mulheres } \\
\%\end{array}$ \\
\hline \multicolumn{10}{|l|}{$\begin{array}{l}\text { Escolaridade } \\
\text { (anos) }\end{array}$} \\
\hline $0-8$ & 2,7 & 1,6 & 3,3 & 1,8 & 1,7 & 1,8 & 0,7 & 0,3 & 1,0 \\
\hline \multicolumn{10}{|l|}{ Estado civil } \\
\hline Conjunto & 13,5 & 18,3 & 10,5 & 8,4 & 10,9 & 6,9 & 3,4 & 2,8 & 3,8 \\
\hline Avulso & 10,6 & 8,1 & 12,1 & 9,5 & 8,9 & 9,8 & 4,9 & 4,5 & 5,2 \\
\hline Ativos $^{1}$ & 24,3 & 26,6 & 22,9 & 9,8 & 10,8 & 9,2 & 2,9 & 2,4 & 3,2 \\
\hline \multicolumn{10}{|l|}{ Estado de saúde } \\
\hline Bom & 16,9 & 18,8 & 15,7 & 12,7 & 14,9 & 11,3 & 5,8 & 5,6 & 5,9 \\
\hline Regular & 6,6 & 7,1 & 6,3 & 4,8 & 4,8 & 4,8 & 2,3 & 1,5 & 2,8 \\
\hline Ruim & 0,6 & 0,5 & 0,7 & 0,5 & 0,4 & 0,4 & 0,3 & 0,2 & 0,4 \\
\hline \multicolumn{10}{|l|}{ Morbidade } \\
\hline Hipertensão & 9,3 & 10,9 & 8,4 & 4,6 & 5,1 & 4,3 & 1,8 & 1,0 & 2,3 \\
\hline Diabetes & 3,4 & 4,3 & 2,9 & 1,3 & 1,4 & 1,1 & 0,6 & 0,3 & 0,7 \\
\hline Dislipidemia & 8,1 & 8,1 & 8,2 & 4,4 & 4,1 & 4,5 & 2,0 & 1,3 & 2,5 \\
\hline \multicolumn{10}{|l|}{ Regiões } \\
\hline Centro-Oeste & 26,6 & 27,7 & 25,7 & 14,2 & 14,8 & 13,7 & 6,0 & 4,9 & 6,6 \\
\hline Sudeste & 23,3 & 27,6 & 20,8 & 23,9 & 26,8 & 22,2 & 8,7 & 7,5 & 9,6 \\
\hline Sul & 25,4 & 29 & 23,3 & 18,1 & 19,7 & 17,2 & 6,9 & 6,1 & 7,3 \\
\hline
\end{tabular}

* Percentual ponderado para ajustar a distribuição sociodemográfica da amostra Vigitel à distribuição da população adulta projetada para a cidade em cada ano analisado.

${ }^{1}$ caminhada + ciclismo; ${ }^{2}$ hipertensão arterial sistêmica; ${ }^{3}$ diabetes; ${ }^{4}$ dislipidemias

A associação da prática de caminhada e do ciclismo foi mais forte para os homens no lazer e no deslocamento ao trabalho; e nas mulheres, no deslocamento à escola. A idade foi associada com maior força para os adultos na faixa de 35 a 64 anos de idade. O estado civil, a percepção do estado de saúde, a obesidade e a distribuição regional dos ativos não evidenciaram associação com a marcha e o ciclismo. Apresentar maior tempo de escolaridade aumentou a razão de chance para a associação da prática de caminhada e de ciclismo em todos os domínios e subdomínios investigados (Tabela 2). 
Tabela 2 - Associações da caminhada e do ciclismo recreativo no deslocamento ativo (trabalho e/ou escola), segundo características individuais e sociodemográficas. Vigitel, 2016.

\begin{tabular}{|c|c|c|c|c|c|c|}
\hline Variáveis & $\begin{array}{c}\text { Lazer } \\
\text { RC (IC 95\%) }\end{array}$ & $\mathrm{p}$ & $\begin{array}{c}\text { Deslocamento ao tra- } \\
\text { balho } \\
\text { RC (IC 95\%) }\end{array}$ & $\mathrm{p}$ & $\begin{array}{c}\text { Deslocamento à escola } \\
\text { RC (IC 95\%) }\end{array}$ & $\mathrm{p}$ \\
\hline \multicolumn{7}{|l|}{ Sexo } \\
\hline Masculino & 1 & & 1 & & 1 & \\
\hline Feminino & $0,92(0,86-0,99)$ & 0,001 & $0,87(0,83-1,0,92)$ & $<0,001$ & $1,72(1,24-2,40)$ & 0,001 \\
\hline \multicolumn{7}{|l|}{ Idade (anos) } \\
\hline $18-34$ & 1 & & 1 & & 1 & \\
\hline $35-54$ & $6,10(5,05-7,36)$ & $<0,001$ & $5,81(4,76-5,75)$ & $<0,001$ & $0,93(0,86-0,98)$ & $<0,001$ \\
\hline $55-64$ & $2,75(2,30-3,29)$ & $<0,001$ & $0,87(0,81-0,97)$ & $<0,001$ & $0,88(0,81-0,93)$ & $<0,001$ \\
\hline$\geq 65$ & $3,93(3,07-4,98)$ & $<0,001$ & $0,83(0,74-0,94)$ & $<0,001$ & $0,77(0,68-0,82)$ & $<0,001$ \\
\hline \multicolumn{7}{|l|}{$\begin{array}{l}\text { Escolaridade } \\
\text { (anos) }\end{array}$} \\
\hline $0-8$ & 1 & & 1 & & 1 & \\
\hline $9-11$ & $1,42(1,32-1,56)$ & $<0,001$ & $1,43(1,35-1,51)$ & $<0,001$ & $1,64(1,38-1,07)$ & 0,094 \\
\hline$\geq 12$ & $1,62(1,51-1,87)$ & 0,003 & $1,65(1,52-1,81)$ & $<0,001$ & $1,82(1,47-1,43)$ & 0,505 \\
\hline \multicolumn{7}{|l|}{ Estado Civil } \\
\hline Par & 1 & & 1 & & 1 & \\
\hline Sozinho & $0,89(0,78-1,09)$ & 0,072 & $0,88(0,76-1,02)$ & 0,100 & $0,49(0,37-0,67)$ & $<0,001$ \\
\hline \multicolumn{7}{|c|}{ Estado de saúde } \\
\hline Bom & 1 & & 1 & & 1 & \\
\hline Regular & $0,68(0,53-1,27)$ & 0,072 & $0,84(0,55-1,28)$ & 0,427 & $0,93(0,63-1,37)$ & 0,729 \\
\hline Ruim & $0,34(0,29-1,05)$ & 0,993 & $0,58(0,49-5,10)$ & 0,441 & $0,79(0,37-5,51)$ & 0,997 \\
\hline \multicolumn{7}{|l|}{ Morbidades } \\
\hline Sim & 1 & & 1 & & 1 & \\
\hline Não & $1,43(1,25-1,65)$ & $<0,001$ & $1,07(0,94-1,23)$ & 0,271 & $1,07(0,78-1,48)$ & 0,637 \\
\hline \multicolumn{7}{|l|}{ Obesidade } \\
\hline Sim & 1 & & 1 & & 1 & \\
\hline Não & $1,16(0,81-1,68)$ & 0,403 & $1,98(0,94-2,29)$ & 0,743 & $2,29(0,92-3,29)$ & 0,380 \\
\hline \multicolumn{7}{|l|}{ Sobrepeso } \\
\hline Sim & 1 & & 1 & & 1 & \\
\hline Não & $1,22(1,08-1,37)$ & 0,001 & $1,52(1,21-1,89)$ & $<0,001$ & $1,33(1,01-1,77)$ & 0,046 \\
\hline \multicolumn{7}{|l|}{ Região } \\
\hline Centro-Oeste & 1 & & 1 & & 1 & \\
\hline Nordeste & $0,96(0,81-1,13)$ & 0,638 & $1,93(0,69-2,25)$ & 0,652 & $1,31(1,02-3,97)$ & 0,034 \\
\hline Norte & $0,82(0,68-0,99)$ & 0,402 & $1,79(0,60-2,05)$ & 0,109 & $1,50(1,09-3,63)$ & $<0,001$ \\
\hline Sudeste & $0,89(0,71-1,12)$ & 0,330 & $2,83(0,97-4,19)$ & 0,317 & $1,28(1,02-4,98)$ & 0,039 \\
\hline Sul & $0,90(0,76-1,05)$ & 0,205 & $1,98(0,82-3,41)$ & 0,558 & $1,14(0,67-2,63)$ & 0,372 \\
\hline
\end{tabular}

1: Referência; RC: Razão de chance (probabilidade de que o evento ocorra dividido pela probabilidade de que o evento não ocorra); IC 95\%: Intervalo de Confiança a 95\%; p: nível de significância (0,05\%). 
O maior percentual de entrevistados $(37,8 \%$ no lazer; $46,0 \%$ no deslocamento para o trabalho e $51,8 \%$ no deslocamento para a escola) relatou caminhar ou pedalar com frequência de 3 a 4 vezes na semana. $\mathrm{O}$ menor percentual de entrevistados (22,9\% no lazer; $13,2 \%$ no deslocamento para o trabalho e $13,9 \%$ no deslocamento para a escola) relatou caminhar ou pedalar com frequência de 1 a 2 vezes na semana (Tabela 3 ).

Tabela 3. Percentual ${ }^{\star}$ de adultos ativos que relataram praticar caminhada e/ou ciclismo recreativo e para o deslocamento ativo (trabalho/escola), por sexo, segundo a frequência semanal. Vigitel, 2016.

\begin{tabular}{lccc}
\hline \multicolumn{1}{c}{ Variáveis } & Total & Homens & Mulheres \\
\hline \multicolumn{1}{c}{ Frequência AF no lazer } & $\%$ & $\%$ & $\%$ \\
\hline a 2 vezes & 22,9 & 23,8 & 22,3 \\
3 a 4 vezes & 37,8 & 37,0 & 38,6 \\
5 a 6 vezes & 27,1 & 23,6 & 29,7 \\
Todos os dias & 12,2 & 15,6 & 9,4 \\
$\quad$ Frequência AF - Trabalho & & & \\
1 a 2 vezes & 13,2 & 12,5 & 13,7 \\
3 a 4 vezes & 46,0 & 43,7 & 47,8 \\
5 a 6 vezes & 28,5 & 29,1 & 28,0 \\
Todos os dias & 12,3 & 14,7 & 10,5 \\
$\quad$ Frequência AF - Escola & & & \\
1 a 2 vezes & 13,9 & 16,1 & 13,0 \\
3 a 4 vezes & 51,8 & 50,8 & 52,2 \\
5 a 6 vezes & 27,7 & 26,9 & 28,1 \\
Todos os dias & 6,6 & 6,2 & 6,7 \\
\hline
\end{tabular}

* Percentual ponderado para ajustar a distribuição sociodemográfica da amostra Vigitel à distribuição da população adulta projetada para a cidade em cada ano analisado.

\section{Discussão}

No Brasil, a caminhada e o ciclismo são praticados largamente por adultos de ambos os sexos. No ano de 2016, a escolha por um desses dois tipos de AF foi a opção de metade da população adulta ativa residente nas capitais dos estados brasileiros e no Distrito Federal. Os homens relataram maior interesse por uma destas duas modalidades no lazer e no deslocamento para o trabalho. As mulheres relataram maior interesse por uma destas duas modalidades no deslocamento para a escola.

O estímulo promotor de marcha e de ciclismo para as mulheres pode ser uma estratégia de sucesso para diminuir as diferenças de prevalência de AF entre os sexos, desfavorável a favor das mulheres. ${ }^{9,10}$. A inatividade física feminina é uma preocupação que, embora seja apenas uma peça em um quadro complexo, pode estar contribuindo para o aumento das condições adversas à saúde das mulheres.

As diferenças na participação em AF entres os sexos podem ser explicadas por interferências de fatores sociodemográficos e ambientais. A falta de tempo em face de dupla jornada de trabalho, a dedicação do tempo livre para o cuidado dos familiares, a insegurança urbana e o medo de eventos adversos à AF são as principais justificativas dadas por elas. A maior prevalência 
de inatividade física feminina parece não ter relação com a falta de informações sobre os benefícios da $\mathrm{AF}$ à saúde ${ }^{11,12}$.

$\mathrm{O}$ envelhecimento está consolidado como um fator negativo associado à aderência para a AF. Está largamente demonstrado o decréscimo da taxa de ativos com o aumento da idade ${ }^{13}$. Neste estudo, a frequência de marchadores e de ciclistas acompanhou essa tendência de diminuição da AF com o aumento da idade. Para os mais idosos, as limitações físicas por problemas crônicos de saúde e a aproximação da aposentadoria - média de 59,4 anos em $2015^{14}$ - pode explicar, em parte, a diminuição da AF recreativa e no deslocamento para o trabalho, respectivamente.

Alguns estudos apontam relação negativa da caminhada e do ciclismo como meio de transporte utilizado para ir ao trabalho. Hardman et al. ${ }^{15}$, em 2013, avaliaram 1.910 industriários no estado de Pernambuco e verificaram que $84,2 \%$ desses trabalhadores se deslocavam passivamente ao trabalho. Silva et al. ${ }^{16}$, em 2011, avaliaram 2.265 trabalhadores gaúchos e mostraram $13,7 \%$ de homens e $12,8 \%$ de mulheres ativos no deslocamento para o trabalho. Neste estudo, em 2016, para o conjunto das 27 cidades avaliadas pelo Vigitel, $32 \%$ da amostra referiram deslocamento ativo. Neste estudo, em 2016, para o conjunto das 27 cidades avaliadas pelo Vigitel, 32\% da amostra referiram deslocamento ativo, representando o dobro de ativos em comparação com os resultados de Silva et. al.

A maior frequência verificada neste estudo do deslocamento ativo para a escola entre os mais jovens (18-34 anos de idade) se justifica pelo encerramento do ciclo escolar por volta dos 35 anos de idade. Segundo dados do Censo da Educação Superior de 2015, a média de idade dos alunos de 100 cursos superiores no Brasil apresentou variação de idade de 22,3 anos (curso de Ciências e Tecnologia) a 36,8 anos (curso de Teologia $)^{17}$. De modo adicional, a percepção da insegurança pública tem provocado alterações significativas na escolha dos modos de transporte, desfavorecendo os deslocamentos urbanos a pé ou de bicicleta ${ }^{18 ; 19}$.

No Brasil, algumas intervenções recentes para promover o transporte ativo têm sido propostas, como a instauração da visão de rede de transporte ativo integrado com o transporte coletivo, a obtenção de dados para monitoramento e fiscalização do deslocamento ativo, a instituição de diretrizes regulamentadas de infraestrutura urbana, o direcionamento de investimentos de infraestrutura, a estimulação e incentivo do uso do transporte ativo, entre outras iniciativas (WRI Brasil, 2017) ${ }^{19}$.

Nos Estados Unidos, em 2015, o National Center for Safe Routes to School, associado ao Departamento de Transporte daquele país, criou um projeto nomeado de Walking School Bus, o qual busca incentivar o transporte ativo de escolares. $\mathrm{O}$ projeto incentiva grupos de crianças a irem a pé à escola, acompanhados de um ou mais adultos conhecidos. Uma variação daquele projeto foi o Bicycle Train, em que adultos supervisionam crianças que usam a bicicleta para irem até suas instituições de ensino ${ }^{20}$.

Tanto as iniciativas brasileiras quanto as estadunidenses carecem, até o momento, de estudos de impacto para demonstrar a efetividade da introdução dessas novas medidas. Nesse sentido, parece necessário conhecer a parcela da população com baixo índice de deslocamento ativo, de modo a fornecer a esse público uma mensagem incentivadora direcionada e acessível.

O maior tempo de escolaridade aumentou a chance de o estudante se deslocar ativamente para a escola. Em estudo com amostra semelhante, foram analisadas, no ano de 2014, informações de 52.779 adultos residentes nas capitais brasileiras ${ }^{21}$, mostrando maior nível de AF com o aumento da escolaridade $(\mathrm{p} \leq 0,001)$. Ainda no mesmo estudo, $46 \%$ dos participantes com maior escolaridade alcançaram a meta de AF recomendada pela OMS. Nota-se que, na década que separa aquele estudo deste, o percentual de ativos que alcançou a meta de AF referindo a caminhada e o ciclismo como atividade principal foi símile $(46 \%$ e $45 \%$, respectivamente).

O estado civil só evidenciou associação para o deslocamento à escola. Viver acompanhado aumentou a chance de se deslocar à escola ativamente. Essa observação encontra apoio nos resultados de Proper e Hildebrandt ${ }^{22}$, os quais mostraram que, na Holanda, casados são pessoas com maior idade que solteiros e optam frequentemente pela marcha e pelo ciclismo como 
forma de AF. Solteiros, normalmente mais jovens, mostraram preferência por práticas de atividades mais vigorosas.

Este estudo mostrou que a avaliação negativa do estado de saúde não se associou com a participação de caminhada e de ciclismo, corroborando com os resultados da análise sistematizada de estudos publicados entre 2005 a 2009, conduzida por Vancea et al. ${ }^{23}$, no qual pessoas mais ativas autoavaliaram a própria saúde de forma mais otimista. Adicionalmente, Cardoso et al. ${ }^{24}$ avaliaram idosos com média de idade de 70 anos e evidenciaram maior grau de satisfação com o estado de saúde para os idosos mais ativos.

Os portadores de morbidades crônicas não transmissíveis (hipertensão, diabetes $\mathrm{e}$ dislipidemias) apresentaram menor chance de caminhar ou pedalar recreativamente quando comparado a seus pares isentos de morbidades. Esse resultado se mostra desafiador à Atenção Primária da Saúde no sentido de reforçar a comunicação aos doentes crônicos dos efeitos substanciais à saúde promovido pela $\mathrm{AF}$ regular. Nesse sentido, como exemplo ilustrativo, podese citar o destaque nas diretrizes de AF da British Heart Foundation $(\mathrm{BHF})^{25}$ sobre os benefícios da AF como fator de proteção às doenças crônicas não transmissíveis. Adicionalmente às diretrizes do BHF, duas metanálises envolvendo publicações abrangendo 737.588 participantes, ampararam a atualização das diretrizes de AF oficiais canadenses de $2010^{26}$, destacando à população o efeito protetor da AF para a hipertensão arterial e diabetes. No mesmo sentido, a prática de caminhada e do ciclismo mostraram-se eficientes para o controle e prevenção das epidemias, enquanto a inatividade física se apresentou deletéria ${ }^{27}$. A plausibilidade biológica tem sido atribuída, em parte, à ação da enzima muscular lipoproteína lipase que aumenta a degradação das moléculas de gordura do sangue, ativada pelo esforço físico e pela diminuição da secreção de triglicerídeos no fígado ${ }^{28}$.

Este estudo mostrou ser maior a chance de adultos sem sobrepeso caminhar ou pedalar. Para o transporte ativo e para a recreação, essa associação apresentou-se na direção esperada, ou seja, quanto menor o peso da massa corporal, mais fácil ser transportado e mais fácil de se movimentar. Uma revisão sistemática da literatura foi realizada com dados de 2010 a 2011, incluindo 95 artigos, evidenciando forte associação na direção esperada (transporte mais ativo associado com menor peso corporal $)^{29}$. A participação de AF com o intuito de perder peso, embora seja real, não ficou explícita nesses resultados.

Nesta investigação, não foi detectado um padrão linear de associação para a prática de caminhada e de ciclismo nas diferentes regiões do país. A comparação deste estudo com outros similares ficou prejudicada pela escassez de amplitude da amostra. No entanto, parece prudente destacar que as cidades de pequeno e médio porte do interior do país podem apresentar comportamentos distintos deste estudo. Assim, as estratégias incentivadoras para a prática de caminhada e de ciclismo poderiam ser pensadas considerando o porte populacional das cidades.

Parece pacificado que a distribuição do volume da AF ao longo da semana é a condição mais desejosa para a saúde. A plausibilidade biológica se sustenta no princípio de as mudanças morfofuncionais do organismo serem mediadas pelos processos de adaptação funcional em resposta ao esforço físico e volta aos padrões anteriores à medida que o intervalo entre os estímulos se alonga ${ }^{30}$. Desse modo, os resultados deste estudo mostraram a maior parte dos ativos com frequência semanal igual ou superior a três vezes, alinhando-se às recomendações. A reduzida frequência semanal da AF constituiu um fator limitante para o não alcance das metas de AF. Do mesmo modo, o estudo conduzido por Lima et al. ${ }^{21}$, em 2014, classificou o nível da AF de 52.779 adultos residentes nas capitais brasileiras e mostrou $95 \%$ de subativos com frequência da AF de 1 e 2 vezes por semana. A simulação de aumento de uma única sessão por semana, elevou o nível de $85 \%$ do grupo subativo para ativo.

A determinação da frequência semanal ideal de $\mathrm{AF}$ ainda não é uma discussão encerrada. $\mathrm{O}$ Comitê Consultivo do Departamento de Saúde dos Estados Unidos (DHHS) ${ }^{4}$, em 2008, entendeu que as orientações do Colégio Americano de Medicina Esportiva, de 2007, eram muito específicas ao definir a frequência mínima de 5 dias por semana para atividades moderadas e 3 dias para intensidades vigorosas. Como resultado, as atuais orientações do DHHS, seguidas pela 
OMS, orientam que se possa acumular AF sem uma frequência semanal definida. A esse respeito, ainda é limitado o número de publicações de estudos de intervenção bem controlada que avaliam os benefícios da AF para a saúde em resposta a uma diminuta frequência semanal das atividades.

Algumas limitações deste estudo devem ser consideradas na interpretação de seus resultados. Os dados do Vigitel foram obtidos exclusivamente por entrevistas de pessoas com acesso à linha de telefone fixo. O uso de pesos pós-estratificados procurou minimizar possíveis diferenças entre a população total e a população analisada. Contudo, existe a possibilidade de viés de seleção residual. No inquérito do Vigitel, o deslocamento a pé ou de bicicleta não foi diferenciado. Assim, não foi possível isolar a participação de cada possibilidade. Outro aspecto que influencia a qualidade das estimativas de inquérito semelhantes ao Vigitel é a taxa de sucesso $(68,5 \%)$ e de recusas $(3,8 \%)$. Nos dois casos o desempenho do Vigitel, em 2016, foi superior à média observada no sistema equivalente dos Estados Unidos (Behavioral Risk Factor Surveillance System) ${ }^{30}$. Relatar AF depende da capacidade de uma pessoa de retornar informações precisas e pode estar sujeita a viés de memória ou desejo social. Para minimizar os efeitos de viés de memória, as perguntas referentes à prática da $\mathrm{AF}$ se limitaram aos três meses anteriores à data da entrevista, sendo as informações submetidas a um Controle de Qualidade com acompanhamento aleatório das entrevistas por um supervisor. Para minimizar o desejo social de supervalorização da AF, o sistema obteve forte reprodutibilidade e acurados em estudos preocupados com a validade dos indicadores de AF e sedentarismo. ${ }^{31}$

Os pontos fortes deste estudo encontram-se nas informações sobre a prática de caminhada $\mathrm{e}$ de ciclismo de numerosa amostra de brasileiros adultos residentes nas capitais dos estados e no Distrito Federal, onde habitam aproximadamente $25 \%$ da população brasileira, periodicamente monitorada pelo sistema Vigitel, desde o ano de 2006. Seus resultados representam evidências relevantes sobre a participação de brasileiros adultos com atividades de marcha e de ciclismo recreativo e no deslocamento ativo.

\section{Conclusão}

Este trabalho se concentrou em associar, a partir de informações sociodemográficas e pessoais, a prática da atividade física recreativa e do deslocamento ativo com a caminhada e o ciclismo de brasileiros adultos. Inicialmente, a amostra foi grande o suficiente para ter um interesse para os subgrupos populacionais que vivem em outros grandes centros urbanos brasileiros. As análises sobre os fatores associados destacaram as associações homogêneas e heterogêneas de acordo com o domínio estudado.

Foi mostrado que homens, na faixa etária de 35 a 54 anos, com maior escolaridade, que vivem acompanhados, que não apresentam doenças crônicas e sobrepeso apresentam maior chance de participar de caminhadas ou de ciclismo recreativo no deslocamento ativo. Os resultados reforçaram a ideia de que a promoção da participação nessas duas modalidades de AF requer atingir a população certa com mensagem correta. Especificamente para os deslocamentos ativos, seria interessante entender o fluxo da prática ou da não prática de caminhar ou pedalar para o trabalho ou para a escola. Saber a proporção de indivíduos que não têm aptidão para caminhar ou pedalar para o trabalho ou para a escola em relação àqueles que têm a possibilidade, mas optam por não fazê-lo, colocaria em perspectiva interessante a mensagem de saúde pública encorajando essas práticas.

\section{Referências}

1. Organização Mundial da Saúde [homepage na internet]. Physical activity for health: More active people for a healthier world: draft global action plan on physical activity 2018-2030. [acesso 23 jul 2018]. Disponível em: http://apps.who.int/gb/ebwha/pdf_files/WHA71/ A71_18-en.pdf

2. Organização das Nações Unidas [homepage na internet]. Revision of World Urbanization Prospects 2018. [acesso 23 set 2018]. Disponível em: https://www.un.org/ development/desa/publications/2018-revision-of-worldurbanization-prospects.htm 
3. Russ LB, Webster CA, Beets MW, Phillips DS. Systematic Review and Meta-Analysis of Multi-Component Interventions Through Schools to Increase Physical Activity. Journal of Physical Activity and Health 2015;12(10): 1436-1446.

4. Department of Health and Human Services (USA). Physical Activity Guidelines for Americans, 2nd ed. Washington (DC): U.S. Department of Health and Human Services; 2018.

5. American College of Sport Medicine. ACMS Guidelines for Exercise Testing and prescription. 8th ed. Philadelphia: Williams \& Wilkins; 2007.

6. Lima DF, Levy RB, Luiz OC. Recomendações para atividade física e saúde: consensos, controvérsias e ambiguidades. Rev Panam Salud Publica. 2014;36(3):16470 .

7. Brasil. Ministério da Saúde. Secretaria de Vigilância em Saúde. Departamento de Análise em Saúde e Vigilância de Doenças não Transmissíveis. Vigitel - Brasil 2016: Vigilância de Fatores de Risco e Proteção para Doenças Crônicas por Inquérito Telefônico. Brasília (DF): Ministério da Saúde; 2017.

8. World Health Organization [homepage na internet]. Epidemic of obesity and overweight linked to increased food energy supply - study. July 2015. [Acesso $03 \mathrm{fev}$ 2018]. Disponível em: http://www.who.int/bulletin/ releases/NFM0715/en/

9. Telford RM, Telford RD, Olive LS, Cochrane T, Davey R. Why Are Girls Less Physically Active than Boys? Findings from the LOOK Longitudinal Study. PLoS One 2016;11(3):e0150041.

10. Hands B, Parker H, Larkin D, Cantell M, Rose E. Male and Female Differences in Health Benefits Derived from Physical Activity: Implications for Exercise Prescription. J Womens Health, Issues Care 2016; 5:4.

11. Edwards ES, Sackett SC. Psychosocial Variables Related to Why Women are Less Active than Men and Related Health Implications. Supplementary Issue: Health Disparities in Women. First Published 2016;4(9)(Suppl 1):47-56.

12. Lima DF, Piovani VGSP, Lima LA. Prática de futebol recreativo entre adultos residentes nas capitais brasileiras, 2011-2015. Epidemiol. Serv. Saúde 2018; 27(2):1-10.

13. Hallal PC. Atividade física e saúde no Brasil: pesquisa, vigilância e políticas. Cad. Saúde Pública 2014;30(12):2487-2489.

14. Instituto Brasileiro de Geografia e Estatística (IBGE). Síntese de indicadores sociais: uma análise das condições de vida da população brasileira: 2016/IBGE, Coordenação de População e Indicadores Sociais. Rio de Janeiro: IBGE; 2016.
15. Hardman CM, Barros SSH, Oliveira ESA, Nahas MV, Barros MVG. Inatividade nos deslocamentos para o trabalho e fatores associados em industriários. Saúde Soc. São Paulo 2013;22(3):760-772, 2013.

16. Silva SG, Duca GF, Silva KSS, Oliveira ESA, Nahas MV. Deslocamento para o trabalho e fatores associados em industriários do sul do Brasil. Rev. Saúde Pública 2012;46(1):180-184.

17. Instituto Nacional de Estudos e Pesquisas Educacionais Anísio Teixeira. Resumo técnico: Censo da Educação Superior 2015. 2. ed. Brasília (DF); 2018.

18. Lima DF, Lima LA; Luiz, OC. Daily physical activity of Brazilian carriers of arterial hypertension: a transversal analysis. Colombia Médica 2017;48(2): 82-87.

19. WRI Brasil [homepage na internet]. 20 ações para impulsionar o transporte ativo no Brasil. [acesso 23 jul 2018]. Disponível em: https://wribrasil.atavist.com/20acoes-para-fomentar-o-transporte-ativo-no-brasil.

20. National Center for Safe Routes to School. The Walking School Bus: Combining Safety, Fun and the Walk to School. North Carolina (USA). University of North Carolina Highway Safety Research Center; 2009.

21. Lima DF, Lima LA, Mazzardo O, Anguera MG, Piovani VGS, Junior APS, et al. O padrão da atividade física no lazer de brasileiros idosos. Caderno de Educação Física e Esporte 2018;16(2):39-49. |

22. Proper KI, Hildebrandt VH. Physical activity among Dutch workers: differences between occupations. Prev Med 2006;43:42-45.

23. Vancea LA, Barbosa JMV, Menezes AS, Santos CM. Associação entre atividade física e percepção de saúde em adolescentes: revisão sistemática. Revista Brasileira de Atividade Física \& Saúde 2011;16(3):246-54.

24. Cardoso AS, Mazo GZ, Salin MS, Santos CAX. Percepção subjetiva de saúde e nível de atividade física de idosos Rev. Bras. Geriatr. Gerontol 2008;11(1):81-91.

25. British Heart Foundation (UK). Physical activity and your heart. London. BHF; 2013.

26. Kesaniemi A, Riddoch CJ, Reeder B, Blair SN, Sorensen TI. Advancing the future of physical activity guidelines in Canada: an independent expert panel interpretation of the evidence. International Journal of Behavioral Nutrition and Physical Activity 2010;7:41-54.

27. Pereira LP, Sichieri R, Segri NJ, Silva RMVG, Ferreira MG. Self-reported dyslipidemia in central-west Brazil: prevalence and associated factors. Ciência \& Saúde Coletiva 2015; 20(6): 1815-1824. 
28. Gill JM, Hardman AE. Exercise and postprandial lipid metabolism: an update on potential mechanisms and interactions with high-carbohydrate diets (review). J Nutr Biochem 2003;14(3):122-32.

29. Aoyagi Y, Park H, Park S, Shephard RJ. Habitual physical activity and health-related quality of life in older adults: interactions between the amount and intensity of activity. Qual Life Res 2010;19(3): 333-338.

30. Centers for Desiase Control and Prevention [homepage na internet]. Behavioral Risk Factor Surveillance System. [acesso 14 ago 2018]. Disponível em: https://www.cdc. gov/brfss/

31. Monteiro CA, Florindo AA, Claro RM, Moura EC. Validade de indicadores de atividade física e sedentarismo obtidos por inquérito telefônico. Rev. Saúde Pública 2008;42(4):575-81.

\section{Como citar este artigo:}

Lima DF, Lima LA, Mazzardo Júnior o, Sampaio AA, Silva MP, Silva Júnior AP, Piovani VGS, Anguera MG. Associação da atividade física de lazer e do deslocamento ocupacional com a caminhada e o ciclismo: um estudo transversal com brasileiros adultos. Rev. Aten. Saúde. 2019; 17(62): 40-51. 